\title{
Effective thermal conductivities of heterogeneous media containing multiple imperfectly bonded inclusions
}

\author{
H. L. Duan and B. L. Karihaloo \\ School of Engineering, Cardiff University, Queen's Buildings, The Parade, Cardiff CF24 3AA, United Kingdom
}

(Received 17 August 2006; revised manuscript received 1 December 2006; published 13 February 2007)

In a recent paper [Duan et al., Phys. Rev. B 73, 174203 (2006)], we derived explicit expressions for the effective conductivities of heterogeneous media containing perfectly bonded ellipsoidal inclusions of diverse shapes, spatial distributions, and orientations. In this paper, we take into account the effect of three types of imperfect bonding between the inclusions and the matrix by replacing the imperfectly bonded ellipsoidal inclusions with equivalent perfectly bonded homogeneous inclusions using the average $t$-matrix approximation of the multiple-scattering approach. The explicit expressions remain unaltered in form but involve the parameters of the equivalent homogeneous inclusions. It is shown that our approximate scheme gives very accurate predictions of the effective conductivity of the heterogeneous materials, while retaining the simplicity of the explicit expressions. However, in contrast to the perfectly bonded inclusions, the effective conductivities of a heterogeneous medium containing imperfectly bonded inclusions depend upon the size of the inclusions. This size dependence is shown to be captured by simple scaling laws depending upon the type of bond imperfection.

DOI: 10.1103/PhysRevB.75.064206

PACS number(s): 72.80.Tm, 05.60.Cd, 44.10.+i, 77.84.Lf

\section{INTRODUCTION}

The effect of imperfect interfaces between the inclusions and the surrounding matrix on the conductivities of a heterogeneous medium is often captured by using a low conducting (LC), a high conducting (HC) interface model of zero thickness, or an interphase model of finite thickness. ${ }^{1,2}$ The LC interface is also called an interface with Kapitza thermal resistance. ${ }^{3}$ The effect of LC interface on the effective conductivities of heterogeneous media has been studied since the 1970s. ${ }^{4-12}$ For example, Hasselman and Johnson ${ }^{7}$ extended the classical work of Maxwell and Rayleigh to derive effective medium approximations (EMAs) for the effective thermal conductivity of heterogeneous media containing spherical particles or cylindrical fibers. Nan et al. ${ }^{11}$ developed a more general EMA formulation for the effective thermal conductivity of a two-phase heterogeneous medium containing spheroidal inclusions with interfacial thermal resistance by exploiting the multiple-scattering approach. Duschlbauer et al. ${ }^{12}$ analyzed the effect of an imperfect thermal resistance between matrix and inclusion based on the idea of alternately replacing the inclusion and the thermal barrier by an equivalent inclusion with a perfect interface and lesser conductivity. The effect of an HC-type bond imperfection on the effective conductivities of heterogeneous media has been studied by Miloh and Benveniste, ${ }^{1}$ Torquato and Rintoul, ${ }^{10}$ Cheng and Torquato, ${ }^{13}$ and Lipton, ${ }^{14}$ among others.

The effective properties of a heterogeneous medium are affected by four factors, namely, the locations (spatial distribution), orientations and shapes of the inclusions, and the interface bonding condition. Some work has been reported on the effect of two or three of these four factors on the effective conductivities. For example, Nan et al. ${ }^{11}$ took into account three factors, namely, the shapes of inclusions (spheroids), their orientations, and the interface bonding condition. In a recent paper, ${ }^{15}$ we have derived explicit expressions for the effective conductivities of heterogeneous media containing perfectly bonded ellipsoidal inclusions of diverse shapes, spatial distributions, and orientations. To the authors' best knowledge, at present, there is no scheme available to predict the joint effect of all the above four factors on the effective conductivities of heterogeneous media.

In the present paper, we will take into account the effect of three types of imperfect bonding identified above by replacing the imperfectly bonded ellipsoidal inclusions with equivalent perfectly bonded homogeneous inclusions using the average $t$-matrix approximation of the multiple-scattering approach. The explicit expressions derived in our recent paper ${ }^{15}$ remain unaltered in form but now involve the parameters of the equivalent homogeneous inclusions. Thus, the present scheme takes into account all the four microstructural parameters that characterize the locations (spatial distribution), orientations and shapes of the inclusions, and the interface bonding condition. It is shown that compared with the available numerical results, the conductivity tensor of the equivalent inclusion together with the explicit expressions in the paper $^{15}$ give very accurate predictions of the effective conductivity of the heterogeneous materials, while retaining the simplicity of these explicit expressions. However, in contrast to the perfectly bonded inclusions, the effective conductivities of a heterogeneous medium containing inclusions with LC- or HC-type bond imperfection depend upon the size of the inclusions. It is shown that this size dependence can be captured by simple scaling laws depending upon the type of bond imperfection.

\section{FORMULATION}

For a representative volume element (RVE) of an ergodic heterogeneous medium with volume $V$, let $\mathbf{q}(\mathbf{x})$ denote the local heat flux at position, $\mathbf{x}, \mathbf{H}(\mathbf{x})$ the local intensity field, and $\Phi(\mathbf{x})$ the temperature field. The local heat flux and intensity fields within $V$ can be obtained by solving the so-called local problem described by the following basic equations: 


$$
\begin{gathered}
\boldsymbol{\nabla} \cdot \mathbf{q}(\mathbf{x})=0, \quad \boldsymbol{\nabla} \times \mathbf{H}(\mathbf{x})=0, \quad \mathbf{H}(\mathbf{x})=-\boldsymbol{\nabla} \Phi(\mathbf{x}), \\
\mathbf{q}(\mathbf{x})=\mu(\mathbf{x}) \cdot \mathbf{H}(\mathbf{x}), \quad \text { or } \mathbf{H}(\mathbf{x})=\zeta(\mathbf{x}) \cdot \mathbf{q}(\mathbf{x}),
\end{gathered}
$$

where $\mu(\mathbf{x})$ and $\zeta(\mathbf{x})$ are the second order conductivity and resistivity tensors, respectively. Generally, the boundary conditions on the boundary $\partial \mathrm{V}$ of the RVE can be specified in one of the following two equivalent forms:

$$
\Phi(\mathbf{x})=-\mathbf{H}^{0} \cdot \mathbf{x} \text { or } \mathbf{q}(\mathbf{x}) \cdot \mathbf{M}(\mathbf{x})=\mathbf{q}^{0} \cdot \mathbf{M}(\mathbf{x}),
$$

where $\mathbf{x}$ is the position vector, $\mathbf{H}^{0}$ and $\mathbf{q}^{0}$ are the constant intensity and heat flux, respectively, and $\mathbf{M}(\mathbf{x})$ is the unit vector normal to the boundary $\partial \mathrm{V}$.

In order to solve the boundary-value problem, apart from Eqs. (1) and (2) for each of the constituents (i.e., the inclusions and the matrix), the interface conditions between the constituents must be prescribed. Below, we will summarize the interface conditions for the three types of bond imperfection identified above.

(a) Low conducting interface $(L C)$. The temperature field is discontinuous across the interface, but the normal component of the heat flux is continuous. The LC-type bond imperfection simulates a thin interphase with low conductivity. The LC interface conditions are

$$
\left[q_{n}\right]=0, \quad[\Phi]=-\alpha q_{n},
$$

where $q_{n}$ is the normal component of the heat flux, $[\cdots]$ $=$ (out) - (in) represents the discontinuity of a quantity across the interface, and $\alpha$ is an interface parameter; $\alpha \rightarrow 0$ denotes an ideal interface, whereas $\alpha \rightarrow \infty$ denotes adiabatic contact.

(b) High conducting interface ( $\mathrm{HC}$ ). The temperature field is continuous across the interface, but the normal component of the heat flux is not. The HC-type bond imperfection simulates a thin interphase with high conductivity. The HC interface conditions are

$$
[\Phi]=0, \quad\left[q_{n}\right]=\beta \Delta_{S} \Phi,
$$

where $\Delta_{S}$ is the surface Laplacian of $\Phi .{ }^{1} \beta \rightarrow 0$ denotes an ideal interface, whereas $\beta \rightarrow \infty$ describes contact with a medium of infinite conductivity.

(c) Interphase model. The LC- and HC-type bond imperfections are both two-phase models in the sense that the interface regions occupy no volume in the heterogeneous medium, whereas the interphase model is a three-phase one consisting of the inclusion, the interphase of finite thickness, and the matrix. In the interphase model, perfect bonding conditions are usually assumed to prevail at both the inclusion/ interphase interface $\Gamma_{I c}$ and the interphase/matrix interface $\Gamma_{c 1}$, i.e.,

$$
\left[\Phi^{k}\right]=0, \quad\left[q_{n}^{k}\right]=0 \quad(k=1,2),
$$

where the superscript $k=1,2$ represents the interfaces $\Gamma_{I c}$ and $\Gamma_{c 1}$, respectively. $\left[\Phi^{k}\right]$ and $\left[q_{n}^{k}\right]$ represent the discontinuities in the temperature and normal heat flux at the interfaces $\Gamma_{I c}$ and $\Gamma_{c 1}$.

\section{CONDUCTIVITIES WHEN THE INCLUSIONS ARE IMPERFECTLY BONDED}

The presence of bond imperfections between the ellipsoidal inclusions and matrix considerably complicates the mathematical analysis, and it is difficult to find an analytical solution of the boundary-value problem described by Eqs. (1)-(4), or (5). Miloh and Benveniste ${ }^{1}$ used ellipsoidal harmonic functions to solve the boundary-value problem with the HC-type interface, but the resulting expressions for the effective conductivities are very complicated and thus of limited usefulness. Therefore, in order to reveal the effect of the bond imperfection on the conductivity properties and to obtain explicit expressions which are easy to use we take an alternative approach based on the concept of an equivalent inclusion, i.e., a fictitious inclusion perfectly bonded to the matrix. The key problem is to obtain the conductivity of the equivalent inclusion corresponding to each of the three types of bond imperfection. Once this key problem is solved we can use all the expressions obtained in our earlier paper. ${ }^{15}$

We begin by calculating the conductivity tensor $\mu_{e}$ of the equivalent inclusion corresponding to the ellipsoidal inclusion with an interphase of finite thickness between it and the matrix. We can then deduce by an appropriate limiting process the conductivity tensors $\mu_{e}$ of equivalent inclusions corresponding to the LC- and HC-type bond imperfections.

\section{A. Conductivities of equivalent inclusions}

Let us regard an ellipsoidal inclusion with conductivity tensor $\mu_{r}$ surrounded by a thin interphase layer of constant thickness $t$ and conductivity tensor $\mu_{c}$ as an equivalent inclusion. It should be noted that the assumption of constant interphase thickness for an arbitrary ellipsoid is only valid when the thickness is infinitesimally small. The ellipsoidal inclusion and ellipsoidal interphase have the same center $O$, and have the same axes. The semiaxes $a_{r}, b_{r}$, and $c_{r}$ of ellipsoidal inclusion are aligned with the axes of $z, x$, and $y$ of the Cartesian coordinate systems, respectively. The conductivity tensor of this equivalent inclusion can be obtained using the average $t$-matrix approximation (ATA) of the multiplescattering approach. ${ }^{16,17}$ In particular, if both the inclusion and the interphase constituents of this equivalent inclusion are isotropic, then the components of its conductivity tensor $\mu_{e}$ can be shown to be

$$
\mu_{i e}=\mu_{c}+\frac{f_{c}\left(\mu_{r}-\mu_{c}\right) \mu_{c}}{\mu_{c}+S_{i r}\left(\mu_{r}-\mu_{c}\right)\left(1-f_{c}\right)},
$$

where $i=x, y, z$ refers to the coordinate axes, $\mu_{r}$ is the conductivity of the isotropic ellipsoidal inclusion, and $\mu_{c}$ is the conductivity of its surrounding isotropic interphase. Here, $f_{c}=a_{r} b_{r} c_{r} /\left(a_{r}+t\right)\left(b_{r}+t\right)\left(c_{r}+t\right)$, where $a_{r}, b_{r}$, and $c_{r}$ are the semiaxes of the ellipsoidal inclusion, and $S_{i r}$ is the component of the geometrical tensor of the ellipsoidal inclusion given in the appendix of Duan et al. ${ }^{15}$ In theory, the above equation is approximately valid for thin interphase layers only. In practice, as will be shown below by numerical computations, the results for such layers are surprisingly accurate. 
We now pass to the limit as $t \rightarrow 0$ and $\mu_{c} \rightarrow 0$, i.e., we regard the interface thermal resistance as the limiting case of heat conduction across the bulk phase separated by an extremely thin, poorly conducting interphase layer. As a result we obtain the conductivity tensor components $\mu_{i e}$ of the equivalent ellipsoidal inclusion corresponding to the LC-type bond imperfection

$$
\mu_{i e}=\frac{\mu_{r}}{1+\alpha \mu_{r} S_{i r}\left(\frac{1}{a_{r}}+\frac{1}{b_{r}}+\frac{1}{c_{r}}\right)},
$$

where $\alpha \equiv \lim _{t \rightarrow 0}\left(t / \mu_{c}\right)$. For the case of a spheroidal $\mu_{c} \rightarrow 0$

inclusion $\left(b_{r}=c_{r}, \gamma_{r}=b_{r} / a_{r}\right)$, Eq. (7) reduces to

$$
\mu_{L e}=\frac{\mu_{r}}{1+\frac{\alpha \mu_{r} S_{L r}\left(\gamma_{r}+2\right)}{b_{r}}}, \quad \mu_{T e}=\frac{\mu_{r}}{1+\frac{\alpha \mu_{r} S_{T r}\left(\gamma_{r}+2\right)}{b_{r}}},
$$

where $\mu_{L e}=\mu_{z e}$ and $\mu_{T e}=\mu_{x e}=\mu_{y e}$.

At the other extreme, when the normal heat flux experiences a jump across the interface, we pass to the limit as $t$ $\rightarrow 0$ and $\mu_{c} \rightarrow \infty$, and obtain the conductivity tensor components of the equivalent ellipsoidal inclusion corresponding to the HC-type bond imperfection

$$
\mu_{i e}=\mu_{r}+\beta\left(1-S_{i r}\right)\left(\frac{1}{a_{r}}+\frac{1}{b_{r}}+\frac{1}{c_{r}}\right),
$$

where $\beta \equiv \lim _{\substack{t \rightarrow 0 \\ \mu_{c} \rightarrow \infty}}\left(\mu_{c} t\right)$. For the case of a spheroidal inclusion, Eq. (9) reduces to

$$
\mu_{L e}=\mu_{r}+\beta\left(1-S_{L r}\right) \frac{\gamma_{r}+2}{b_{r}}, \quad \mu_{T e}=\mu_{r}+\beta\left(1-S_{T r}\right) \frac{\gamma_{r}+2}{b_{r}} .
$$

Equations (8) and (10) are identical to those of Nan et al. ${ }^{11}$ Additional special cases are the following. If $\gamma_{r}=1, \mu_{L e}$ and $\mu_{T e}$ in Eqs. (8) and (10) reduce to those for spherical inclusions. If $\gamma_{r} \rightarrow 0, \mu_{T e}$ in Eqs. (8) and (10) reduce to those for cylindrical fibers. Finally, if $\gamma_{r} \rightarrow \infty$, then $\mu_{T e}$ in Eqs. (8) and (10) reduces to that for penny sheets.

It should be emphasized that although we deduced the conductivities [Eqs. (7)-(10)] of the equivalent inclusions corresponding to the LC- and HC-type bond imperfections by limiting processes from the conductivities [Eq. (6)] of the equivalent ellipsoidal inclusion corresponding to the interphase-type bond imperfection, the definitions of the two interface parameters $\alpha$ and $\beta$ are not limited to $\alpha$ $\equiv \lim _{\substack{t \rightarrow 0 \\ \mu_{c} \rightarrow 0}}\left(t / \mu_{c}\right)$ and $\beta \equiv \lim _{\substack{t \rightarrow 0 \\ \mu_{c} \rightarrow \infty}}\left(\mu_{c} t\right)$. Other definitions are also possible. For example, the Kapitza resistance $R_{d}(=\alpha)$ can be measured at different temperatures by measuring the ratio of the temperature drop to the heat flux across an interface. ${ }^{10}$

\section{B. Accuracy of the equivalent inclusion approximation}

We will take the three-phase configuration of a spheroidal inclusion with an interphase in an infinite matrix as an example to solve the local fields exactly. The results of the LCand HC-type bond imperfections can be obtained by limiting processes from those of the interphase-type bond imperfection. We will then use the exact local fields of a spheroidal inclusion with an interphase in an infinite matrix subjected to a uniform far field intensity and other known numerical results to establish the accuracy of the intensities obtained by the present approximate scheme [Eqs. (6)-(10)]. The three regions, namely, the inclusion, the interphase and the matrix, are isotropic and homogeneous and are labeled $r, c$, and 1 . The two interfaces between $r$ and $c$, and between $c$ and 1 are denoted by $L_{1}$ and $L_{2}$, respectively. The outer $L_{2}$ and inner $L_{1}$ spheroidal surfaces have a common center $O$ and axis of revolution $(\mathrm{Oz})$, and they are not allowed to intersect. The axes of $x, y$ and $z$ of the Cartesian coordinate system are aligned with the semiaxes of $L_{1}$ and $L_{2}$. The isotropic conductivities of the inclusion, interphase and matrix are denoted by $\mu_{r}, \mu_{c}$, and $\mu_{1}$, respectively. In general, the position of $L_{2}$ relative to $L_{1}$ can be different in this three-phase configuration, e.g., the interphase can be confocal when $L_{1}$ and $L_{2}$ have the same foci, it can be equithick when the interphase thicknesses along the minor and major axes $x, y$, and $z$ of $L_{1}$ and $L_{2}$ are equal, or it can be homothetic when $L_{1}$ and $L_{2}$ are homothetic. For these three relative positions of $L_{1}$ and $L_{2}$, the local fields (intensity and flux) of the three-phase configuration are different, but they can be obtained by procedures similar to that described in the Appendix.

In order to examine the accuracy of the intensities obtained by the present approximate scheme and by Duschlbauer et al., ${ }^{12}$ we consider the case of equithickness interphase and compare the temperature gradient concentration tensors $D_{i j}^{r}$ (defined as $H_{i}^{r}=D_{i j}^{r} H_{j}^{0}$ ) in the inclusion and $D_{i j}^{m}$ (defined as $H_{i}^{m}=D_{i j}^{m} H_{j}^{0}$ ) in the matrix, where $i, j=x, y, z, H_{i}^{r}$ and $H_{i}^{m}$ are the intensities in the inclusion and matrix, respectively, and $H_{i}^{0}$ is the intensity at infinity. For a spheroidal inclusion with an equithickness interphase in an infinite matrix under a uniform far field intensity, the local fields in the inclusion are not constant. We calculate the components $D_{z z}^{r}$ and $D_{z z}^{m}$ of the intensity tensors in the inclusion and matrix under the only nonvanishing remote intensity $H_{z}^{0}$. For the three-phase configuration under the remote axisymmetric intensity $H_{z}^{0}$, the temperature fields in Eq. (A5) of the Appendix can be simplified by letting $m=0$, because the temperature fields are symmetrical with respect to the $z$ axis.

Let us now compare the numerical results obtained by the different approximate schemes with the exact solution for the case of the LC interface. The intensities of the LC-type bond imperfection can be obtained by a limiting process from those of the interphase-type bond imperfection, namely, $t$ $\ll a_{r}$ and $\mu_{c} \ll \mu_{r}$. Therefore, the parameters for the numerical computations are chosen as $a_{r}=2 \mu \mathrm{m}, \quad \alpha=6$ $\times 10^{-8} \mathrm{~W} / \mathrm{m}^{2} \mathrm{~K}, \quad t=0.3 \mathrm{~nm}, \quad \mu_{c}=0.005 \mathrm{~W} / \mathrm{mK}, \quad \mu_{r}$ $=600 \mathrm{~W} / \mathrm{mK}, \mu_{1}=17.4 \mathrm{~W} / \mathrm{mK}$, and $\gamma=5$. Without reproducing the tedious calculations here, the exact solutions of $D_{z z}^{r}$ and $D_{z z}^{m}$ can be obtained following the procedure of the Appendix, and the results for the chosen parameters are plot- 


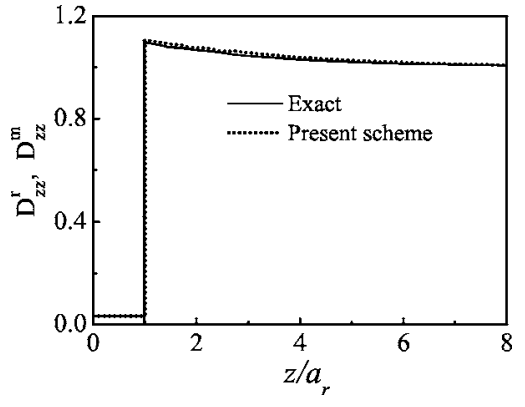

FIG. 1. Comparison of the exact temperature gradient concentration tensors $D_{i j}^{r}$ and $D_{i j}^{m}$ with those obtained the present approximate scheme.

ted in Fig. 1 together with the results predicted the present approximate scheme. It is clear from Fig. 1 that the predictions of present scheme are very close to the exact results both in the inclusion and the matrix.

Duschlbauer et al. ${ }^{12}$ gave the numerical results obtained by their approximate averaging scheme and the finite element method for the volume average $\bar{D}_{z z}^{r}$ in the inclusion. For the chosen parameters, $\bar{D}_{z z}^{r}=0.0312$ by their approximate averaging scheme, and $\bar{D}_{z z}^{r}=0.0310$ by finite element method. The exact result is $\bar{D}_{z z}^{r}=0.0317$, and that by the present scheme is $\bar{D}_{z z}^{r}=0.0324$. Thus, for the spheroid with a general aspect ratio the results obtained by the present approximate scheme [Eq. (10)] are very close to the exact results and to those of Duschlbauer et al. ${ }^{12}$ Without reproducing the details here, we have also confirmed that for inclusions in the shape of spheres, disks, and cylinders, the results obtained by the present scheme are identical to the exact results and to the results of Duschlbauer et al. ${ }^{12}$ The approximate averaging scheme and the finite element method in the paper of Duschlbauer et al., ${ }^{12}$ furnish results in a numerical form; by contrast the expressions obtained by the present approximate scheme are in closed form, easy to use, and just as accurate. Moreover, in Sec. V, we will show that compared with the numerical results of Miloh and Benveniste, ${ }^{1}$ the conductivity tensor [Eqs. (6)-(10)] of the equivalent inclusion together with the corresponding scheme for effective conductivity of the heterogeneous materials give very accurate predictions for the effective conductivity of the heterogeneous materials under consideration, and the predictive formula is again in a simple closed form.

\section{Heterogeneous medium with diverse spatial distributions and orientations of imperfectly bonded inclusions}

For an ergodic heterogeneous medium consisting of ellipsoidal inclusions of $N-1$ different types in a homogeneous matrix, the matrix will be denoted by the superscript 1 , and its conductivity tensor and volume fraction by $\boldsymbol{\mu}_{1}$ and $f_{1}$, respectively. The inclusions with conductivity tensor $\boldsymbol{\mu}_{r}$ and the volume fraction $f_{r}$ may differ in size, shape, orientation and the type of bond imperfection. The effective conductivity tensor $\boldsymbol{\mu}$ of such an $N$-phase heterogeneous medium is still given by Eqs. (30), (31) in the paper of Duan et al., ${ }^{15}$ but with $\boldsymbol{\mu}_{r}$ in Eq. (31) replaced by $\boldsymbol{\mu}_{e}$ of Eq. (6), (7), or (9) depending on the type of bond imperfection.

In what follows, we will therefore present, without detail, the effective conductivities of only those heterogeneous media containing inclusions with diverse orientational distributions and bond imperfections for which the results can be compared with those available in the literature. This comparison will provide further validation of our approximate approach based on the concept of the equivalent inclusion. It will also help in revealing how the effective conductivities in the presence of bond imperfections differ from their counterparts when the inclusions are perfectly bonded to the matrix.

With these two aims in mind, consider an orientational distribution of spheroidal inclusions that is intermediate between the random and the aligned ones, for example, the distribution with the probability density function $D(\theta, \varphi)$ $\left(=\frac{1}{2 \pi}\left[\left(\Lambda^{2}+1\right) e^{-\Lambda \theta}+\Lambda e^{-\Lambda \pi / 2}\right]\right)$, where $\theta$ and $\varphi$ describe the orientation of a spheroidal inclusion. ${ }^{15}$ The effective conductivities of the heterogeneous medium with this distribution of spheroidal inclusions are ${ }^{15}$

$$
\begin{gathered}
\mu_{T}=\mu_{1}+\mu_{1}\left[\left(\left\{k_{1 r}\right\}+R_{1}\left\{k_{2 r}\right\}\right)^{-1}-S_{T V}\right]^{-1}, \\
\mu_{L}=\mu_{1}+\mu_{1}\left[\left(\left\{k_{1 r}\right\}+R_{2}\left\{k_{2 r}\right\}\right)^{-1}-S_{L V}\right]^{-1}
\end{gathered}
$$

in which

$$
\begin{gathered}
R_{1}=\frac{18-\Lambda\left(\Lambda^{2}+3\right) e^{-\Lambda \pi / 2}}{6\left(\Lambda^{2}+9\right)} \\
R_{2}=\frac{\left(\Lambda^{2}+3\right)\left(3+\Lambda e^{-\Lambda \pi / 2}\right)}{3\left(\Lambda^{2}+9\right)}, \quad\left\{k_{1 r}\right\}=\sum_{r=2}^{N} k_{1 r}, \quad\left\{k_{2 r}\right\}=\sum_{r=2}^{N} k_{2 r} \\
k_{1 r}=f_{r}\left(\frac{\mu_{1}}{\mu_{T r}-\mu_{1}}+S_{T r}\right)^{-1}, \\
k_{2 r}=f_{r}\left[\left(\frac{\mu_{1}}{\mu_{L r}-\mu_{1}}+S_{L r}\right)^{-1}-\left(\frac{\mu_{1}}{\mu_{T r}-\mu_{1}}+S_{T r}\right)^{-1}\right]
\end{gathered}
$$

where $S_{L r}, S_{T r}, S_{L V}$, and $S_{T V}$ are the components of $\mathbf{S}_{r}$ and $\mathbf{S}_{V}$ of the spheroidal inclusion and distribution spheroid, respectively, given in the appendix of Duan et al. ${ }^{15}$ but with $\mu_{L r}$ and $\mu_{T r}$ replaced by $\mu_{L e}$ and $\mu_{T e}$ given by Eqs. (8) and (10) depending on the type of bond imperfection.

As shown in Duan et al. ${ }^{15}$ the probability density function $D(\theta, \varphi)$ can cover several orientations of inclusions: (a) the random orientation, which corresponds to $\Lambda=0, R_{1}=R_{2}$ $=1 / 3$; (b) the aligned orientation, which corresponds to $\Lambda$ $=\infty, R_{1}=0, R_{2}=1$; (c) the slightly perturbed aligned orientations, corresponding to a large value of $\Lambda$, with $R_{1}$ and $R_{2}$ in Eq. (12) being $R_{1} \simeq 3 / \Lambda^{2}, R_{2} \simeq 1-6 / \Lambda^{2}$; (d) the weakly expressed orientational preference, corresponding to a small value of $\Lambda$, with $R_{1}$ and $R_{2}$ in Eq. (12) being $R_{1} \simeq 1 / 3$ $-\Lambda / 18, R_{2}=1 / 3+\Lambda / 9$.

(a) Random orientation $(\Lambda=0)$. For a two-phase heterogeneous medium containing randomly oriented spheroidal inclusions with imperfect bonding, the shape of the distribution ellipsoid is a sphere, the conductivity tensor is isotropic, so that Eqs. (11) and (12) reduce to 


$$
\mu_{T}=\mu_{L}=\mu_{1}+\mu_{1} \frac{3 f_{2}\left(2 \xi_{T}+\xi_{L}\right)}{9-f_{2}\left(2 \xi_{T}+\xi_{L}\right)}
$$

in which

$$
\xi_{T}=\frac{\mu_{T e}-\mu_{1}}{\mu_{1}+S_{T 2}\left(\mu_{T e}-\mu_{1}\right)}, \quad \xi_{L}=\frac{\mu_{L e}-\mu_{1}}{\mu_{1}+S_{L 2}\left(\mu_{L e}-\mu_{1}\right)} .
$$

Equations (13) and (14) are not identical to those [Eq. (23)] of Nan et al. ${ }^{11}$ This is because Nan et al. ${ }^{11}$ used the MoriTanaka averaging scheme ${ }^{18}$ which, for random orientation of inclusions, does not coincide with the averaging scheme used in our earlier paper, ${ }^{15}$ except for spherical inclusions. The accuracy of our averaging scheme ${ }^{15}$ has been established for the perfectly bonded ellipsoidal inclusions with random orientation, for which the predictions of the counterparts of Eqs. (13) and (14) agree very well with experimental data. We shall return to the numerical comparison between our results and those of Nan et al. ${ }^{11}$ later in the paper.

(b) Aligned orientation $(\Lambda=\infty)$. For a two-phase heterogeneous medium containing aligned spheroidal inclusions with imperfect bonds, the shape of the distribution is a spheroid, and Eqs. (11) and (12) reduce to

$$
\mu_{T}=\mu_{1}+\mu_{1} \frac{f_{2} \xi_{T}}{1-f_{2} \xi_{T} S_{T V}}, \quad \mu_{L}=\mu_{1}+\mu_{1} \frac{f_{2} \xi_{L}}{1-f_{2} \xi_{L} S_{L V}} .
$$

When $S_{T V}=S_{T 2}$ and $S_{L V}=S_{L 2}$, the above expressions reduce to the results obtained by the Mori-Tanaka scheme. ${ }^{18}$ When $S_{T V}=S_{T 2}=1 / 2$ and $S_{L V}=S_{L 2}=0$, the result for a two-phase heterogeneous medium containing aligned cylindrical fibers coincides with that of Nan et al. ${ }^{11}$ [their Eq. (18)].

(c) Slightly perturbed aligned orientations. For a twophase heterogeneous medium containing spheroidal inclusions with slightly perturbed aligned orientations and imperfect bonds, the shape of the distribution ellipsoid is a spheroid, and Eqs. (11) and (12) reduce to

$$
\begin{aligned}
& \mu_{T}=\mu_{1}+\mu_{1} \frac{f_{2}\left[3 \xi_{L}+\left(\Lambda^{2}-3\right) \xi_{T}\right]}{\Lambda^{2}-f_{2} S_{T V}\left[3 \xi_{L}+\left(\Lambda^{2}-3\right) \xi_{T}\right]}, \\
& \mu_{L}=\mu_{1}+\mu_{1} \frac{f_{2}\left[\left(\Lambda^{2}-6\right) \xi_{L}+6 \xi_{T}\right]}{\Lambda^{2}-f_{2} S_{L V}\left[\left(\Lambda^{2}-6\right) \xi_{L}+6 \xi_{T}\right]} .
\end{aligned}
$$

(d) Weakly expressed orientational preference. For a twophase heterogeneous medium containing spheroidal inclusions with a weakly expressed orientational preference and imperfect bonds, the shape of the distribution ellipsoid is a spheroid, and Eqs. (11) and (12) reduce to

$$
\begin{aligned}
& \mu_{T}=\mu_{1}+\mu_{1} \frac{f_{2}\left[(12+\Lambda) \xi_{T}-(\Lambda-6) \xi_{L}\right]}{18-f_{2} S_{T V}\left[(12+\Lambda) \xi_{T}-(\Lambda-6) \xi_{L}\right]}, \\
& \mu_{L}=\mu_{1}+\mu_{1} f_{2}\left[(3+\Lambda) \xi_{L}-(\Lambda-6) \xi_{T}\right] / 9-f_{2} S_{L V}\left[(3+\Lambda) \xi_{L}-(\Lambda\right. \\
& \left.-6) \xi_{T}\right] .
\end{aligned}
$$

\section{SCALING LAWS FOR SIZE-DEPENDENCE OF CONDUCTIVITIES}

From the derivation of the conductivity tensors of equivalent inclusions corresponding to the LC- and HC-type bond imperfections two intrinsic length scales automatically emerged, namely,

$$
\begin{aligned}
& l_{\text {low }}=\alpha \mu_{1} \quad \text { for LC-type imperfection, } \\
& l_{\text {high }}=\frac{\beta}{\mu_{1}} \text { for HC-type imperfection. }
\end{aligned}
$$

Dimensional analysis shows that the nondimensional effective conductivities of heterogeneous media containing inclusions with imperfect interfaces of the LC and HC types must depend upon the size of the inclusions. This size dependence is important for the characterization of such heterogeneous media. The detailed size dependence of the effective conductivities can of course be investigated by a close scrutiny of the corresponding expressions which can be rather time consuming. However, as will be shown below, when the intrinsic length scales are small compared with the characteristic size of the heterogeneous medium, the size dependence is accurately captured by simple scaling laws depending on the type of bond imperfection. For the purposes of illustration, we only consider a two-phase heterogeneous medium containing spheroidal inclusions of diverse orientations but with LC or HC type of bond imperfection. The size dependence of the effective conductivities of multiphase heterogeneous media with the same types of bond imperfection also obeys similar scaling laws.

\section{A. Scaling law for LC-type bond imperfection}

The nondimensional effective conductivity of a heterogeneous medium with LC-type imperfect bond can be expressed as a function of the nondimensional parameter $l_{\text {low }} / L$, where $L$ is the characteristic size of the medium. We can expand the expressions for the components of the effective conductivity tensor in a Taylor series in the variable $l_{\text {low }} / L$. When this variable is small so that the terms of the order two and higher can be neglected, these expressions take the following simple form:

$$
\frac{H_{q}(\infty)}{H_{q}(L)}=1+\frac{1}{L} \Upsilon_{l q} l_{\text {low }} \quad(q=T, L),
$$

where $Y_{l q}(\mathrm{q}=\mathrm{T}, \mathrm{L})$ are two nondimensional parameters. Here and in the following, the subscript $q=T, L$ denotes quantities in the transverse and longitudinal directions, respectively. (The subscript $L$ for longitudinal should not be confused with the characteristic length $L) . H_{q}(L)$ denotes a generic conductivity component corresponding to the characteristic size $L$, and $H_{q}(\infty)$ denotes the same property when $L \rightarrow \infty$ or, equivalently, when the effect of bond imperfection is vanishingly small. For the two-phase heterogeneous medium containing spheroidal inclusions, the radius $R$ $=\left(a_{2} b_{2}^{2}\right)^{1 / 3}$ of the equivalent spherical inclusion, which has the same volume as the spheroidal inclusion, is chosen as the characteristic size $L$. Nan and Birringer ${ }^{19}$ have also obtained 
a simple scaling law similar to that in Eq. (20) for the thermal conductivity of polycrystals.

For the two-phase heterogeneous medium containing spheroidal inclusions with LC type of bond imperfection, the two nondimensional parameters $Y_{l T}$ and $Y_{l L}$ in the transverse and longitudinal directions are, respectively,

$$
\begin{aligned}
& \Upsilon_{l T}=f_{2} \Gamma_{2}^{2}\left(\gamma_{2}+2\right) \gamma_{2}^{-1 / 3} \frac{S_{T 2} \eta_{1}-g_{1}\left(R_{1}\right)}{g_{2}\left(R_{1}, S_{T V}\right) g_{2}\left(R_{1}, 1+S_{T V}\right)}, \\
& \Upsilon_{l L}=f_{2} \Gamma_{2}^{2}\left(\gamma_{2}+2\right) \gamma_{2}^{-1 / 3} \frac{S_{T 2} \eta_{1}-g_{1}\left(R_{2}\right)}{g_{2}\left(R_{2}, S_{L V}\right) g_{2}\left(R_{2}, 1+S_{L V}\right)}
\end{aligned}
$$

in which $\Gamma_{2}=\mu_{2} / \mu_{1}$ and

$$
\begin{gathered}
\eta_{1}=\left[\Gamma_{2}-2\left(\Gamma_{2}-1\right) S_{T 2}\right]^{2}, \\
g_{1}(x)=\left(S_{T 2}-S_{L 2}\right)\left[1-\left(1-\Gamma_{2}\right)^{2} S_{L 2} S_{T 2}\right] x, \\
g_{2}(x, y)=f_{2}\left(\Gamma_{2}-1\right)^{2}\left(S_{L 2}-S_{T 2}\right)(1-y) x-\left(2 S_{T 2}+\Gamma_{2} S_{L 2}\right)[1 \\
\left.+\left(\Gamma_{2}-1\right)\left(f_{2}+S_{T 2}-f_{2} y\right)\right] .
\end{gathered}
$$

Note that $\Upsilon_{l T}, \Upsilon_{l L}, H_{q}(\infty)$, and $H_{q}(L)$ are dependent on the orientations (namely, on the parameters $R_{1}$ and $R_{2}$ ) and the distribution (namely, on the parameters $S_{T V}$ and $S_{L V}$ ) of the inclusions. Therefore, the parameters $R_{1}$ and $R_{2}$ in Eqs. (20)-(22) have different values for the four different orientations considered above. Also, $S_{T V}=S_{L V}=1 / 3$ for the random orientation, and $S_{T V}=S_{T 2}$ and $S_{L V}=S_{L 2}$ for the other three orientations (the aligned, the slightly perturbed aligned, and the weakly expressed orientational preference). A comparison of the numerical results from the scaling law in Eqs. (20)-(22) with the exact results from the governing expressions shows that the scaling law Eqs. (20)-(22) is accurate when $l_{\text {low }} \leqslant 0.1 R$ for prolate inclusions and $l_{\text {low }} \leqslant 0.05 R$ for oblate inclusions. In particular, for a two-phase heterogeneous medium containing spherical inclusions, $R_{1}=R_{2}=1 / 3$, $\gamma_{2}=1, S_{T 2}=S_{T V}=1 / 3$. In this case, $\Upsilon_{l T}$ and $\Upsilon_{l L}$ are equal to, say, $\Upsilon_{l s}$

$$
\Upsilon_{l s}=\frac{9 f_{2} \Gamma_{2}^{2}}{\left[\left(1+2 f_{2}\right) \Gamma_{2}+2\left(1-f_{2}\right)\right]\left[\left(1-f_{2}\right) \Gamma_{2}+2+f_{2}\right]} .
$$

A comparison of the scaling law in Eqs. (20) and (23) with the exact results from the governing expressions shows that it is accurate when $l_{\text {low }} \leqslant 0.2 R$, which is a slightly larger range of accuracy than for spheroidal inclusions. For a twophase heterogeneous medium containing cylindrical fibers $R_{1}=0, R_{2}=1, \gamma_{2}=0, S_{T 2}=S_{T V}=1 / 2$, the nondimensional parameter $Y_{l f}$ in the transverse direction is

$$
\Upsilon_{l f}=\frac{4 f_{2} \Gamma_{2}^{2}}{\left[\left(1+f_{2}\right) \Gamma_{2}+1-f_{2}\right]\left[\left(1-f_{2}\right) \Gamma_{2}+1+f_{2}\right]} .
$$

Again, a comparison shows that the scaling law for the effective conductivity in the transverse direction [Eqs. (20) and (24)] is accurate when $l_{\text {low }} \leqslant 0.35 R$. Note that the effective conductivity in the longitudinal direction is simply $\mu_{L}$ $=f_{2} \mu_{2}+\left(1-f_{2}\right) \mu_{1}$, which shows that it is unaffected by the bond imperfection. ${ }^{11}$

\section{B. Scaling law for HC-type bond imperfection}

Following the same procedure as in the preceding section, the size dependence of the effective conductivities of heterogeneous media with HC-type bond imperfection interface model can be shown to obey the scaling law

$$
\frac{H_{q}(L)}{H_{q}(\infty)}=1+\frac{1}{L} \Upsilon_{h q} l_{\text {high }} \quad(q=T, L),
$$

where $\Upsilon_{h q}(\mathrm{q}=\mathrm{T}, \mathrm{L})$ are two nondimensional parameters. For a two-phase heterogeneous medium containing spheroidal inclusions with HC-type bond imperfection, $Y_{h T}$ and $Y_{h L}$, in the transverse and longitudinal directions are, respectively,

$$
\begin{aligned}
& \Upsilon_{h T}=f_{2}\left(\gamma_{2}+2\right) \gamma_{2}^{-1 / 3} \frac{\left(1-S_{T 2}\right) \eta_{1}-g_{3}\left(R_{1}\right)}{g_{2}\left(R_{1}, S_{T V}\right) g_{2}\left(R_{1}, 1+S_{T V}\right)}, \\
& \Upsilon_{h L}=f_{2}\left(\gamma_{2}+2\right) \gamma_{2}^{-1 / 3} \frac{\left(1-S_{T 2}\right) \eta_{1}-g_{3}\left(R_{2}\right)}{g_{2}\left(R_{2}, S_{L V}\right) g_{2}\left(R_{2}, 1+S_{L V}\right)}
\end{aligned}
$$

in which

$$
g_{3}(x)=\left(S_{L 2}-S_{T 2}\right)\left[\Gamma_{2}^{2}-2\left(1-\Gamma_{2}\right)^{2} S_{T 2}\left(1-S_{T 2}\right)\right] x .
$$

$R_{1}, R_{2}, S_{T V}$, and $S_{L V}$ are identical to those for the LC-type bond imperfection. A comparison of the predictions of the scaling law in Eqs. (25) and (26) with the results from the exact expressions for the effective conductivities shows that the above scaling law is accurate when $l_{\text {high }} \leqslant 0.1 R$ for prolate inclusions and $l_{\text {high }} \leqslant 0.05 R$ for oblate inclusions. For a two-phase heterogeneous medium containing spherical inclusions $Y_{h s}=2 \Upsilon_{l s} / \Gamma_{2}^{2}$ [with $\Upsilon_{l s}$ given by Eq. (23)], and scaling law is accurate when $l_{\text {high }} \leqslant 0.2 R$. For a two-phase heterogeneous medium containing cylindrical fibers, the nondimensional parameter $\Upsilon_{h f}$ in transverse direction is $\Upsilon_{h f}=\Upsilon_{l f} / \Gamma_{2}^{2}$ [with $\Upsilon_{l f}$ given by Eq. (24)], and scaling law for the effective conductivity in the transverse direction [Eq. (25)] is accurate when $l_{\text {high }} \leqslant 0.35 R$. As before, there is no size effect in the effective conductivity in the longitudinal direction. It should be pointed out that Wang et al. ${ }^{20}$ have shown that the size dependence of the effective moduli and the Eshelby tensors of the nanostructured materials also follows a scaling law similar to Eq. (25).

\section{NUMERICAL RESULTS}

Let us compare the effective longitudinal conductivity of a two-phase heterogeneous medium containing aligned prolate spheroidal inclusions with the HC-type bond imperfection predicted by Miloh and Benveniste ${ }^{1}$ who solved the boundary-value problem using ellipsoidal harmonic functions with the result obtained by the present approximate scheme based on the concept of an equivalent homogeneous inclusion. The shape and material parameters used in the numerical calculations are taken from Fig. 2 in the paper of Miloh and Benveniste, ${ }^{1}$ i.e., $a_{1}=1.02 C, b_{1}=0.201 C, \gamma_{2}$ $=0.1971$, and $\mu_{1}=5 \mu_{2}$, where $2 C$ denotes the distance be- 


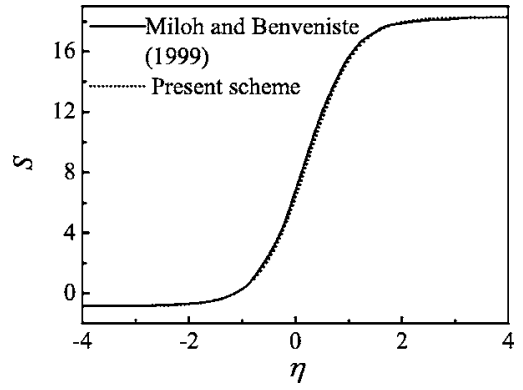

FIG. 2. Comparison of the effective longitudinal conductivity of a two-phase heterogeneous medium containing aligned prolate spheroidal inclusions with HC-type bond imperfection obtained by Miloh and Benveniste (Fig. 2) (Ref. 1) and by the present approximate scheme.

tween the foci of the spheroid. Define two nondimensional parameters $S$ and $\eta$,

$$
S=\frac{1}{f_{2}}\left(\frac{\mu_{L}}{\mu_{1}}-1\right), \quad \eta=\log _{10}\left(\beta C^{-1} \mu_{1}^{-1}\right) .
$$

The variation of $S$ with $\eta$ is shown in Fig. 2. The numerical result of Miloh and Benveniste ${ }^{1}$ is predicted by Eq. (3.37) in their paper, and our numerical results are obtained from Eqs. (10), (14), and (15). It is seen from Fig. 2 that on the scale of the figure the two sets of results are practically indistinguishable, thus further confirming the validity and accuracy of the equivalent homogeneous inclusion concept.

Next, we will compare the effective thermal conductivity of a heterogeneous medium consisting of copper spheroids randomly distributed in an epoxy matrix. The interface between the spheroids and the matrix has low thermal conductivity (Kapitza resistance), i.e., the bond imperfection is of the LC type. The shape parameter of the inclusion and material parameters of the constituents for the numerical calculations are as follows: equivalent radius $R=\left(a_{2} b_{2}^{2}\right)^{1 / 3}$ $=50 \mu \mathrm{m}$, aspect ratio is $\gamma_{2}=0.2$, and $\mu_{2}=10 \mu_{1}$. We choose two values of the interface Kapitza resistance $R_{d}\left(R_{d}=\alpha\right)$ at two different temperatures, namely, $R /\left(\mu_{1} \alpha\right)=14.8$ at $T$ $=4 \mathrm{~K}$ and $R /\left(\mu_{1} \alpha\right)=4.93$ at $T=3 \mathrm{~K} .{ }^{10}$ We compare the results obtained by the present scheme (the exact results and

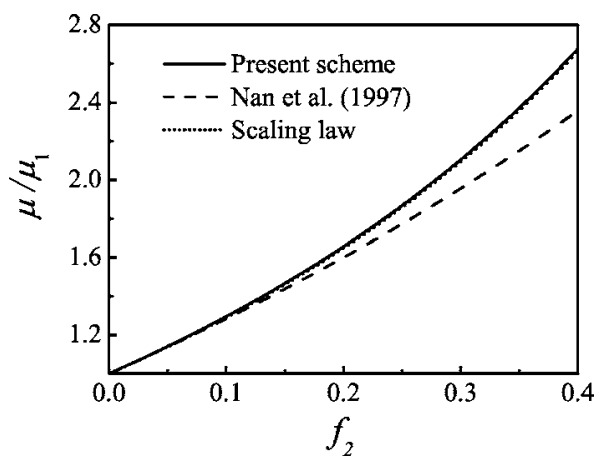

FIG. 3. Effective thermal conductivity of a medium containing copper spheroids randomly distributed in an epoxy matrix and having an LC-type bond imperfection [Kapitza resistance $R /\left(\mu_{1} \alpha\right)$ $=14.8]$.

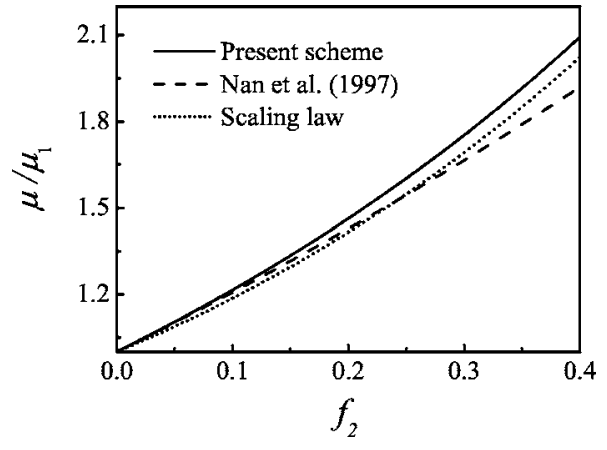

FIG. 4. Effective thermal conductivity of a medium containing copper spheroids randomly distributed in an epoxy matrix and having an LC-type bond imperfection [Kapitza resistance $R /\left(\mu_{1} \alpha\right)$ $=4.93]$.

the results predicted by the scaling law) with those of Nan et al. ${ }^{11}$ The comparison is shown in Figs. 3 and 4. The numerical results of Nan et al. ${ }^{11}$ are predicted using their Eq. (23). Our exact numerical results are obtained from Eqs. (8), (13), and (14), and the approximate results from the scaling law Eqs. (20)-(22). Our results are very close to those of Nan et $a l .{ }^{11}$ for small volume fractions of spheroids and deviate from the latter as the volume fraction increases. Note that when $R /\left(\mu_{1} \alpha\right)=14.8, l_{\text {low }}=0.0676 R<0.1 R$, whereas when $R /\left(\mu_{1} \alpha\right)=4.93, l_{\text {low }}=0.203 R>0.1 R$. We have shown above that the scaling law is accurate for prolate spheroids with LC-type bond imperfection when $l_{\text {low }} \leqslant 0.1 R$. This is borne out by the results shown in Figs. 3 and 4 .

\section{CONCLUSIONS}

We have derived explicit expressions for the effective conductivities of heterogeneous media containing imperfectly bonded ellipsoidal inclusions of diverse shapes, spatial distributions, and orientations. We took into account the effect of three types of imperfect bonding between the inclusions and the matrix by replacing the imperfectly bonded ellipsoidal inclusions with equivalent perfectly bonded homogeneous inclusions using the average $t$-matrix approximation of the multiple-scattering approach. The validity and accuracy of this replacement approximation was demonstrated by comparing the predictions based on it with known numerical results and with the exact analytical results for some special inclusion configurations. These explicit expressions are easy to use. We have shown that, in contrast to the perfectly bonded inclusions, the effective conductivities of a heterogeneous medium containing imperfectly bonded inclusions are dependent upon the size of the inclusions. This size dependence is shown to be captured by simple scaling laws depending upon the type of bond imperfection. The present model is applicable to any type of conduction problem obeying the Laplace equation. It is emphasized that in this paper, we only consider ergodic heterogeneous media, i.e., statistically homogeneous media. The present scheme cannot predict the effective conductivity close to the percolation threshold. 


\section{ACKNOWLEDGMENTS}

Helpful discussions with Jianxiang Wang and assistance with some numerical calculations by Xin Yi, both of Peking University, are gratefully acknowledged. H. L. D. was funded by The Royal Society, London.

\section{APPENDIX}

For the three-phase configuration of a spheroidal inclusion with an interphase in an infinite matrix subjected to a uniform far field intensity, the temperature fields satisfy Laplace's equation. The general solution of Laplace's equation in the oblate spheroidal coordinate system is ${ }^{21}$

$$
\begin{aligned}
\Phi^{i}= & \sum_{n=0}^{\infty} \sum_{m=0}^{n}\left[A_{n}^{i} p_{n}^{m}(\xi)+B_{n}^{i} q_{n}^{m}(\xi)\right] P_{n}^{m}(\eta) \sin m \gamma \\
& +\sum_{n=0}^{\infty} \sum_{m=0}^{n}\left[C_{n}^{i} p_{n}^{m}(\xi)+D_{n}^{i} q_{n}^{m}(\xi)\right] P_{n}^{m}(\eta) \cos m \gamma
\end{aligned}
$$

in which the superscript $i=r, c, 1$ denotes the inclusion, the interphase and the matrix, respectively. $\eta$ and $\xi$ are the auxiliary position parameters for the oblate spheroidal coordinate system $(\alpha, \beta, \gamma)$,

$$
\begin{gathered}
\xi=\sinh \alpha, \quad \bar{\xi}=\cosh \alpha=\sqrt{1+\xi^{2}}, \quad \eta=\cos \beta, \\
\bar{\eta}=\sin \beta=\sqrt{1-\eta^{2}} .
\end{gathered}
$$

$p_{n}^{m}(x)$ and $q_{n}^{m}(x)$ are the modified Legendre functions of order $m$ of the first and second kinds, and are defined as

$$
\begin{gathered}
p_{n}(x)=(-i)^{n} P_{n}(i x), \quad q_{n}(x)=i^{n+1} Q_{n}(i x), \\
p_{n}^{m}(x)=(-i)^{n} P_{n}^{m}(i x), \quad q_{n}^{m}(x)=i^{n+1} Q_{n}^{m}(i x),
\end{gathered}
$$

where $i=\sqrt{-1}, P_{n}^{m}(x)$ and $Q_{n}^{m}(x)$ are the associated Legendre functions of order $m$ of the first and second kinds, and they are defined in the book of Hobson. ${ }^{21}$ As $\alpha \rightarrow \infty, p_{n}^{m}(\xi)$ becomes infinite, so that $A_{n}^{m}=C_{n}^{m}=0$ in the matrix. $q_{n}^{m}(\xi)$ becomes singular as $\alpha=0$, so that $B_{n}^{r}=D_{n}^{r}=0$ in the spheroidal inclusion which contains the origin.

When the spheroidal inclusion and spheroidal interphase do not have common foci (e.g., an equithickness interphase exists between the inclusion and the matrix), two oblate spheroidal coordinate systems " $s$ " and " $e$ " are needed defined by ${ }^{22}$

$$
\begin{aligned}
x & =c_{s} \bar{\xi}_{s} \bar{\eta}_{s} \cos \gamma=c_{e} \bar{\xi}_{e} \bar{\eta}_{e} \cos \gamma, \quad y=c_{s} \bar{\xi}_{s} \bar{\eta}_{s} \sin \gamma \\
& =c_{e} \bar{\xi}_{e} \bar{\eta}_{e} \sin \gamma, \quad z=c_{s} \xi_{s} \eta_{s}=c_{e} \xi_{e} \eta_{e},
\end{aligned}
$$

where $c_{s}$ and $c_{e}$ are the half distances between the foci of the spheroidal inclusion and spheroidal interphase, respectively. The subscripts " $s$ " and " $e$ " represent the parameters in the two coordinate systems, respectively. In such a case, the potentials in Eq. (A1) for the inclusion and interphase are expressed in the oblate coordinate system " $s$," and those for the matrix are expressed in the oblate coordinate system "e." Therefore, the temperature fields in the inclusion, the interphase and the matrix are

$$
\begin{aligned}
\Phi^{r}= & \sum_{n=0}^{\infty} \sum_{m=0}^{n}\left(A_{n}^{r} \sin m \gamma+C_{n}^{r} \cos m \gamma\right) p_{n}^{m}\left(\xi_{s}\right) P_{n}^{m}\left(\eta_{s}\right) \\
\Phi^{c}= & \sum_{n=0}^{\infty} \sum_{m=0}^{n}\left[A_{n}^{c} p_{n}^{m}\left(\xi_{s}\right)+B_{n}^{c} q_{n}^{m}\left(\xi_{s}\right)\right] P_{n}^{m}\left(\eta_{s}\right) \sin m \gamma \\
& +\sum_{n=0}^{\infty} \sum_{m=0}^{n}\left[C_{n}^{c} p_{n}^{m}\left(\xi_{s}\right)+D_{n}^{c} q_{n}^{m}\left(\xi_{s}\right)\right] P_{n}^{m}\left(\eta_{s}\right) \cos m \gamma \\
& \infty \Phi^{1}= \\
& \sum_{n=0} \sum_{m=0}^{n}\left(B_{n}^{1} \sin m \gamma+D_{n}^{1} \cos m \gamma\right) q_{n}^{m}\left(\xi_{e}\right) P_{n}^{m}\left(\eta_{e}\right) \\
& -c_{e}\left(H_{x}^{0} \sin \gamma+H_{y}^{0} \cos \gamma\right) p_{1}^{1}\left(\xi_{e}\right) P_{1}^{1}\left(\eta_{e}\right) \\
& -c_{e} H_{z}^{0} p_{1}\left(\xi_{e}\right) P_{1}\left(\eta_{e}\right),
\end{aligned}
$$

where $A_{n}^{r}, C_{n}^{r}, A_{n}^{c}, B_{n}^{c}, C_{n}^{c}, D_{n}^{c}, B_{n}^{1}$, and $D_{n}^{1}$ are unknown constants to be determined from the following interface and boundary conditions:

$$
\begin{gathered}
\Phi^{r}=\Phi^{c}, \quad \mathbf{q}^{r} \cdot \mathbf{n}_{1}=\mathbf{q}^{c} \cdot \mathbf{n}_{1} \quad \text { at } L_{1}, \\
\Phi^{c}=\Phi^{1}, \quad \mathbf{q}^{c} \cdot \mathbf{n}_{2}=\mathbf{q}^{1} \cdot \mathbf{n}_{2} \quad \text { at } L_{2}, \\
\mathbf{H}^{1}=\mathbf{H}^{0} \quad \text { at infinity. }
\end{gathered}
$$

Here, $\mathbf{n}_{1}$ and $\mathbf{n}_{2}$ are the unit normal vectors to the interfaces between the inclusion and interphase and the interphase and matrix, respectively. To reduce the continuity conditions at interface between the interphase and matrix to equalities between series involving $P_{n}^{m}\left(\eta_{e}\right)$, it is necessary to express $p_{n}^{m}\left(\xi_{s}\right) P_{n}^{m}\left(\eta_{s}\right)$ and $q_{n}^{m}\left(\xi_{s}\right) P_{n}^{m}\left(\eta_{s}\right)$ for the interphase region in the coordinate system " $e$." This operation calls for the expansion formulas for the spheroidal harmonics. The expansion formulas for the spheroidal harmonics can be obtained based on the relation between the Bessel and the Legendre functions. ${ }^{22,23}$ The expansion formulas for the oblate spheroidal harmonics are

$$
P_{m+l}^{m}\left(\eta_{s}\right) q_{m+l}^{m}\left(\xi_{s}\right)=d^{m+l+1} \frac{(2 m+l) !}{l !} \sum_{i=0}^{+\infty}\left\{\sum_{r=0}^{i} \frac{1}{r !}\left[\frac{1-d^{2}}{2}\right]^{r} a_{2 i}^{l+m, r}\right\} \times \frac{(2 i+l) !}{(2 m+2 i+l) !} P_{m+l+2 i}^{m}\left(\eta_{e}\right) q_{m+l+2 i}^{m}\left(\xi_{e}\right),
$$




$$
\begin{aligned}
P_{m+2 l}^{m}\left(\eta_{s}\right) p_{m+2 l}^{m}\left(\xi_{s}\right)=\frac{(2 m+2 l) !}{(2 m+4 l+1)(2 l) !} & \cdot \sum_{k=0}^{l} \frac{(2 m+4 k+1)(2 k) !}{(2 m+2 k) !} \times d^{-(m+2 k)}\left\{\sum_{r=0}^{l-k} \frac{1}{r !}\left[\frac{d^{2}-1}{2 d^{2}}\right]^{r} a_{2 s-2 k}^{m+2 k, r}\right\} P_{m+2 k}^{m}\left(\eta_{e}\right) p_{m+2 k}^{m}\left(\xi_{e}\right), \\
P_{m+2 l+1}^{m}\left(\eta_{s}\right) p_{m+2 l+1}^{m}\left(\xi_{s}\right)= & \frac{(2 m+2 l+1) !}{(2 m+4 l+3)(2 l+1) !} \sum_{k=0}^{l} \frac{(2 m+4 k+3)(2 k+1) !}{(2 m+2 k+1) !} \times d^{-(m+2 k+1)} \\
& \times\left\{\sum_{r=0}^{l-k} \frac{1}{r !}\left[\frac{d^{2}-1}{2 d^{2}}\right]^{r} a_{2 l-2 k}^{m+2 k+1, r}\right\} P_{m+2 k+1}^{m}\left(\eta_{e}\right) p_{m+2 k+1}^{m}\left(\xi_{e}\right)
\end{aligned}
$$

where

$$
a_{2 i}^{n, r}=(-1)^{i+r} C_{i-1}^{i-r}(2 n+4 i+1) \prod_{k=1}^{r-1}[2(n+i+k)+1],
$$

$(1<r \leqslant i), C_{i-1}^{i-r}$ denotes the binomial coefficients, $a_{2 i}^{n, 0}=\delta_{i, 0}$ and $\delta_{i, 0}$ is the Kronecker delta,

$$
a_{2 i}^{n, 1}=(-1)^{i+1}(2 n+4 i+1),(1 \leqslant i), l \geqslant 0, n \geqslant 0, d=c_{s} / c_{e} .
$$

Using the recursion formulas for the associated Legendre functions $P_{n}^{m}(\eta)$, and equating the coefficients of $P_{n}^{m}(\eta)$ and $P_{n}^{m^{\prime}}(\eta)$ in the left and right sides of the interface and boundary conditions (A6), an infinite system of linear algebraic equations for the constants is obtained, where $P_{n}^{m^{\prime}}(\eta)$ is the derivative of $P_{n}^{m}(\eta)$ with respect to $\eta$. In the numerical computations the infinite system is truncated at $n=N$ with $N$ being determined by the requirement that the change from $N$ to $N+1$ does not change the numerical values of the coefficients by more than $10^{-5}$. Note that the local intensity field is not constant in the inclusion when the spheroidal inclusion and spheroidal interphase do not have common foci. However, when they have common foci, only one oblate shperoidal coordinate system is needed. In this case, the temperature fields are still given by Eq. (A5), but the only nonzero constants are $A_{n}^{r}, C_{n}^{r}, A_{n}^{c}, B_{n}^{c}, C_{n}^{c}, D_{n}^{c}, B_{n}^{1}$, and $D_{n}^{1}(n, m=0,1)$. Analytical results of the local fields for the confocal case can be easily obtained, and these fields are constant in the inclusion. These results are the same as those of Bilboul. ${ }^{24}$
*Author to whom correspondence should be addressed. Electronic address: karihaloob@cardiff.ac.uk

${ }^{1}$ T. Miloh and Y. Benveniste, Proc. R. Soc. London, Ser. A 455, 2687 (1999).

${ }^{2}$ Z. Hashin, J. Appl. Phys. 89, 2261 (2001).

${ }^{3}$ P. L. Kapitza, 1941 Collected Papers of P. L. Kapitza, edited by

D. ter Haar (Pergamon, Oxford, 1965), Vol. 2, p. 581.

${ }^{4}$ D. Duschlbauer, H. J. Bohm, and H. E. Pettermann, Mater. Sci. Eng. 19, 1107 (2003).

${ }^{5}$ K. W. Garrett and H. M. Rosenberg, J. Phys. D 7, 1247 (1974).

${ }^{6}$ Y. Benveniste, J. Appl. Phys. 61, 2840 (1987).

${ }^{7}$ D. P. H. Hasselman and L. F. Johnson, J. Compos. Mater. 21, 508 (1987).

${ }^{8}$ A. G. Every, Y. Tzou, D. P. H. Hasselman, and R. Raj, Acta Metall. Mater. 40, 123 (1992).

${ }^{9}$ R. Lipton and B. Vernescu, J. Appl. Phys. 79, 8964 (1996).

${ }^{10}$ S. Torquato and M. D. Rintoul, Phys. Rev. Lett. 75, 4067 (1995).

${ }^{11}$ C. W. Nan, R. Birringer, D. R. Clarke, and H. Gleiter, J. Appl. Phys. 81, 6692 (1997).
${ }^{12}$ D. Duschlbauer, H. E. Pettermann, and H. J. Bohm, J. Appl. Phys. 94, 1539 (2003).

${ }^{13}$ H. Cheng and S. Torquato, Proc. R. Soc. London, Ser. A 453, 1331 (1997).

${ }^{14}$ R. Lipton, J. Mech. Phys. Solids 45, 361 (1997).

${ }^{15}$ H. L. Duan, B. L. Karihaloo, J. Wang, and X. Yi, Phys. Rev. B 73, 174203 (2006).

${ }^{16}$ C. W. Nan, Prog. Mater. Sci. 37, 1 (1993).

${ }^{17}$ C. W. Nan, K. F. Cai, and R. Z. Yuan, Ceram. Int. 22, 457 (1996).

${ }^{18}$ M. L. Dunn and M. Taya, J. Appl. Phys. 73, 1711 (1993).

${ }^{19}$ C. W. Nan and R. Birringer, Phys. Rev. B 57, 8264 (1998).

${ }^{20}$ J. Wang, H. L. Duan, Z. P. Huang, and B. L. Karihaloo, Proc. R. Soc. London, Ser. A 462, 1355 (2006).

${ }^{21}$ E. W. Hobson, The Theory of Spherical and Ellipsoidal Harmonics (Chelsea, New York, 1955).

${ }^{22}$ H. L. Duan, J. Wang, Z. P. Huang, and Y. Zhong, Proc. R. Soc. London, Ser. A 461, 1055 (2005).

${ }^{23}$ A. Riccardi and F. Montheillet, Acta Mech. 133, 39 (1999).

${ }^{24}$ R. R. Bilboul, Br. J. Appl. Phys. 2, 921 (1969). 